\title{
Applications of finite interval-valued hesitant fuzzy preference relations in group decision making
}

\author{
Raúl Pérez-Fernández ${ }^{a}$, Pedro Alonso ${ }^{\mathrm{b}}$, Humberto Bustince ${ }^{\mathrm{c}}$, \\ Irene Díaz ${ }^{\mathrm{d}}$ and Susana Montes ${ }^{\mathrm{a}, *}$ \\ ${ }^{a}$ Department of Statistics and O.R., \\ University of Oviedo. \\ e-mail: \{uo205053,montes\}@uniovi.es \\ ${ }^{\mathrm{b}}$ Department of Mathematics, \\ University of Oviedo. \\ e-mail:palonso@uniovi.es \\ ${ }^{\mathrm{c}}$ Department of Automatics and Computation, \\ Public University of Navarra. \\ e-mail: bustince@unavarra.es \\ ${ }^{\mathrm{d}}$ Department of Computer Science, \\ University of Oviedo \\ e-mail:sirene@uniovi.es
}

\begin{abstract}
The main purpose of this paper is to present the twofold group decision making problem, which is a new point of view of the group decision making problem where several experts and criteria can be considered at the same time. This problem is based on the study of finitely generated sets and finite interval-valued hesitant fuzzy preference relations. Furthermore, the Extended Weighted Voting Method, which is used in the exploitation phase of a classical group decision making problem, is generalized to the twofold case.
\end{abstract}

Key words:

Finite interval-valued hesitant fuzzy preference relation, Finitely generated set, Twofold group decision making problem, Extended Weighted Voting Method.

* Corresponding author. 


\section{Introduction}

In a classical group decision making problem $([6,10])$, some information provided by several experts is considered in order to choose the alternative which is most accepted by these specialists.

However, the experts are not always available to have their experience in different fields. As taking into account at the same time several experts and criteria is very usual, it is a long-standing problem in group decision making. There are several approaches to this problem. Most of them are centred on the concept of hesitant fuzzy sets $([9,21,23,25])$ and the aggregation of this kind of sets $([2,26,27])$. Other authors have proposed using fuzzy preference relations ([14,17,22]), Atanassov's intuitionistic interval fuzzy information ([24]), trapezoidal valued hesitant fuzzy sets ([20]) or hesitant fuzzy linguistic terms ([15]). In all these proposals, several experts are asked for their preference on a set of alternatives considering several criteria. Nevertheless, most of the times, preferences of the same expert in different criteria are treated as preference of different experts in a single-criterion problem. However, in this twofold group decision making problem, preferences of experts in the same criteria are mixed and then this consensus preference is compared with other preferences in different criteria. This consideration is due to the intuitive notion that experts in a same criterion have a similar behaviour. Nevertheless, this method makes sense even though experts in a same criterion actually have different preferences. Moreover, the imprecision and uncertainty caused by the subjectivity in the information provided by the experts could be modelled with this proposal.

In the twofold group decision making problem, finitely generated sets, which are finite unions of closed intervals ([19]), play a fundamental role. This kind of sets allows to construct finite interval-valued hesitant fuzzy preference relations ([19]), which are the measure considered in the twofold case of how much an alternative is preferred to another. However, there are not arithmetic operations or aggregation operators defined between finitely generated sets in the literature. Therefore, a whole section of this paper is devoted to them.

On the other hand, weighted voting method ([3,11-13]) is a common method used to classify the alternatives given a fuzzy preference relation. In [18], we have improved this method in order to construct a family of weighted voting methods depending on a parameter $\alpha$ measuring what is more important: being the "most dominating" or the "least dominated" alternative. This improvement has been called Extended Weighted Voting Method (EWVM for short). However, this proposal can only consider fuzzy preference relations. Therefore, in this paper, this method has been adapted to finite intervalvalued hesitant fuzzy preferences. The resulting method will be henceforth called Twofold Extended Weighted Voting Method (T-EWVM for short). 
The structure of this paper is as follows. First of all a few preliminary definitions are shown in Section 2 in order to fix notations and review several notions in finitely generated sets and finite interval-valued hesitant fuzzy preference relations. Then, some arithmetic operations between finitely generated sets are seen in Section 3 in order to introduce the twofold group decision making problem in Section 4. Then, an illustrative example of the twofold group decision making problem in a practical case is shown in Section 5. Finally, we conclude with some conclusions and open problems.

\section{Preliminary definitions}

This section is devoted to briefly introduce several well-known basic concepts and to fix the notations used in this paper. The study of finitely generated sets, which were firstly considered by Chen et al. [5] and defined in detail by Pérez-Fernández et al. [19], is shortly reviewed right after. Let us start with some notations.

Definition 1 [19] The class of n-finitely generated sets in the interval [0,1] is:

$F G_{n}([0,1])=\left\{J \subseteq[0,1] \mid J=I_{1} \cup \ldots \cup I_{n}\right.$ for some disjoint $\left.I_{1}, \ldots, I_{n} \in L([0,1])\right\}$

where $L([0,1])$ is the set of all closed subintervals in $[0,1]$.

Definition 2 [19] The class of finitely generated sets in the interval [0,1] is:

$$
F G([0,1])=\bigcup_{n=1}^{+\infty} F G_{n}([0,1])
$$

Remark 3 Finitely generated sets can also be defined on any other interval, but in this paper we are mostly working with membership functions and, therefore, we can reduce to $[0,1]$.

Once these sets have been introduced, the following proposition is straightforward.

Proposition 4 [19] Let $I \subseteq[0,1]$. Then, the following statements are equivalent:

- $I \in F G([0,1])$.

- There exists a unique value $n \geq 1$ such that $I \in F G_{n}([0,1])$.

Thus, for any $I \in F G([0,1])$, the unique value $n \geq 1$ such that $I \in F G_{n}([0,1])$ is denoted by $n_{I}$. 
In [19], Pérez-Fernández et al. go in depth on the analysis of this kind of sets. The most important definitions and results on this paper are reviewed right after. There, the concept of $\alpha^{s g}$ of a finitely generated set was introduced, as a generalization of the $\alpha$-point of an interval. For that, an order under the set $[0,1]^{\{-,+\}}$, which is in fact the set $[0,1] \times\{-,+\}$, was defined. After that, the map $\Upsilon$ was introduced, as a way to determine the selected interval of the finitely generated set where the $\alpha^{s g}$-point will be considered. Let us recall this definition.

Definition 5 [19] Let $\alpha^{s g}$ be an element in $[0,1]^{\{-,+\}}$. The function $\Upsilon$ : $[0,1]^{\{-,+\}} \times \mathbb{N} \rightarrow \mathbb{N}$ is defined in the following way:

$\Upsilon\left(0^{-}, n\right)=\Upsilon\left(0^{+}, n\right)=1$

$\Upsilon\left(\frac{i}{n}^{-}, n\right)=i \forall i \in\{1, \ldots, n-1\}$

$\Upsilon\left(\frac{i}{n}^{+}, n\right)=i+1 \forall i \in\{1, \ldots, n-1\}$,

$\Upsilon\left(1^{-}, n\right)=\Upsilon\left(1^{+}, n\right)=n$

$\Upsilon\left(\alpha^{s g}, n\right)=i \forall \alpha$ satisfying $\frac{i-1}{n}<\alpha<\frac{i}{n}$ when $i \in\{1, \ldots, n\}$.

After that, the concept of $\alpha^{s g}$-point of the finitely generated set was defined as follows.

Definition 6 [19] Let $A=\bigcup_{i=1}^{n_{A}} I_{i}$ be a $n_{A}$-finitely generated set and $\alpha^{s g} \in$ $[0,1]^{\{-,+\}}$. The $\alpha^{s g}$-point of the finitely generated set $A$ is defined as

$$
K_{\alpha^{s g}}(A)=\inf \left(I_{\Upsilon\left(\alpha^{s g}, n_{A}\right)}\right)+\alpha^{\prime}\left(\sup \left(I_{\Upsilon\left(\alpha^{s g}, n_{A}\right)}\right)-\inf \left(I_{\Upsilon\left(\alpha^{s g}, n_{A}\right)}\right)\right),
$$

with $\alpha^{\prime}=n_{A} \cdot \alpha-\Upsilon\left(\alpha^{s g}, n_{A}\right)+1 \in[0,1]$.

How to obtain an $\alpha^{s g}$-point by rescaling other two $\alpha^{s g}$-points in the same interval is shown in the following proposition, where the order between the elements in $[0,1]^{\{-,+\}}$was given by $\alpha^{s g 1}<\beta^{s g 2}$ iff $\alpha<\beta$ or $\alpha=\beta$ and $(s g 1, s g 2)=(-,+)($ see $[19])$.

Proposition 7 [19] Let $A \in F G([0,1])$ and let $I_{A}$ be some of the disjoint intervals which compose $A$. Let $\alpha^{s g 1}, \beta^{s g 2}, \gamma^{s g 3}$ be three elements in $[0,1]^{\{-,+\}}$ such that $\alpha^{s g 1}<\beta^{s g 2}<\gamma^{s g 3}$ and $K_{\alpha^{s g 1}}(A), K_{\beta^{s g 2}}(A), K_{\gamma^{s g 3}}(A) \in I_{A}$. Then,

$$
K_{\beta^{s g 2}}(A)=K_{\alpha^{s g 1}}(A)+\frac{\beta-\alpha}{\gamma-\alpha}\left(K_{\gamma^{s g 3}}(A)-K_{\alpha^{s g 1}}(A)\right)
$$


It is well known that every interval is determined unequivocally by its two ends. Furthermore, finitely generated sets can be characterized by certain $\alpha^{s g_{-}}$ points: the grid points.

Definition 8 Let $n \in \mathbb{N}$. A n-grid is defined as:

$$
G(n)=\left\{\frac{i-1^{+}}{n} \mid i=1, \ldots, n\right\} \bigcup\left\{\frac{i^{-}}{n} \mid i=1, \ldots, n\right\} .
$$

$n$-grids are fundamental in the analysis of finitely generated sets. In the following definitions a characterization of a finitely generated set using a certain $n$-grid is proposed.

Definition 9 Let $A$ be a finitely generated set and $n$ a natural number. The set of $n$-grid points of $A$ is defined as the set of all the $\alpha^{s g}$-points of $A$ for any $\alpha^{s g} \in G(n)$ and it is denoted by $K^{n}(A)$. Thus,

$$
K^{n}(A)=\left\{K_{\alpha^{s g}}(A) \mid \alpha^{s g} \in G(n)\right\} .
$$

Definition 10 Let $A$ be a $n_{A}$-finitely generated set. The $n_{A}$-grid points, $K^{n_{A}}(A)$, are called fundamental points of $A$. Note that the fundamental points of any set $A$ determine unequivocally a $n_{A}$-finitely generated set.

Operating with intervals is possible just considering the two bounds of each interval. In the finitely generated set case, basic arithmetic operations can be made considering the union of two specific $n$-grids.

Definition 11 Let $n, m \in \mathbb{N}$. The $n, m$-grid is defined as:

$$
G(n, m)=G(n) \cup G(m) .
$$

Proposition 12 [19] Let $n_{1}, n_{2}, n \in \mathbb{N}$, then

$$
G\left(n_{1}, n_{2}\right)=G(n) \quad \Leftrightarrow \quad \min \left(n_{1}, n_{2}\right) \mid \max \left(n_{1}, n_{2}\right) \text { and } \max \left(n_{1}, n_{2}\right)=n .
$$

In addition, a weak order between finitely generated sets based on the concept of $\alpha^{s g}$-point was introduced.

Definition 13 [19] Let $A, B \in F G([0,1])$ and a fixed $\alpha^{s g} \in[0,1]^{\{-,+\}}$. Then, a weak order on $F G([0,1])$ is defined as:

$$
A \leq_{\alpha^{s g}} B \quad \Leftrightarrow \quad K_{\alpha^{s g}}(A) \leq K_{\alpha^{s g}}(B) .
$$

We can generalise this proposal for ordering $F G([0,1])$ by means of the lattice order

$$
A \leq_{l o} B \quad \Leftrightarrow \quad K_{\alpha^{s g}}(A) \leq K_{\alpha^{s g}}(B), \quad \forall \alpha^{s g} \in[0,1]^{\{-,+\}}
$$


which was proven in [19] to be an order on $F G([0,1])$.

Finally, the definition of fuzzy preference relation and finite interval-valued hesitant fuzzy preference relation must be reviewed.

Definition 14 [8] Given a finite set of alternatives $\mathcal{A}$, a fuzzy preference relation $R$ is a mapping $R: \mathcal{A} \times \mathcal{A} \rightarrow[0,1]$ such that $R(A, B)+R(B, A)=1$ for any pair of alternatives $A$ and $B$ in $\mathcal{A}$.

This kind of relations are also known in the literature as probabilistic, reciprocal or ipsodual relations, depending on the environment we are working ([1]).

Definition 15 [19] Given a finite set of alternatives $\mathcal{A}$, a finite intervalvalued hesitant fuzzy preference relation $R$ is a mapping $R: \mathcal{A} \times \mathcal{A} \rightarrow$ $F G([0,1])$ such that $R(A, B)$ is symmetric to $R(B, A)$ in relation to the point 0.5 for any pair of alternatives $A$ and $B$ in $\mathcal{A}$.

Remark 16 Note that $R(A, B)$ and $R(B, A)$ are finitely generated sets. Therefore, being symmetric in relation to the point 0.5 would be that:

$$
K_{\alpha^{s g}}(R(A, B))+K_{(1-\alpha)^{s g^{c}}}(R(B, A))=1 \forall \alpha^{s g} \in[0,1]^{\{-,+\}} .
$$

For instance, let $A=[0,0.2] \cup[0.4,0.7] \in F G([0,1])$. Then, we obtain $B$, the symmetric of $A$ in relation to the point 0.5 , in the following way:

Firstly, we know that $B \in F G_{2}([0,1])$ as $A \in F G_{2}([0,1])$. Therefore, it is enough to obtain $K_{\alpha^{s g}}(B)$ for $\alpha^{s g} \in G(2)=\left\{0, \frac{1}{2}^{-}, \frac{1}{2}^{+}, 1\right\}$.

$K_{0}(B)=1-K_{1}(A)=1-0.7=0.3$,

$K_{\frac{1}{2}}-(B)=1-K_{\frac{1}{2}}+(A)=1-0.4=0.6$,

$K_{\frac{1}{2}}+(B)=1-K_{\frac{1}{2}}-(A)=1-0.2=0.8$,

$K_{1}(B)=1-K_{0}(A)=1-0=1$.

Finally,

$$
B=[0.3,0.6] \cup[0.8,1]
$$

\section{$3 \quad$ Finitely generated sets arithmetic}

The main goal of this paper is introducing a new kind of group decision making problem. In this problem, finitely generated sets are considered. Furthermore, 
aggregation operators between them are also used. Unfortunately, these operators are not previously defined and, therefore, we need to address their introduction in this section.

\subsection{Basic operations between finitely generated sets}

In order to define aggregation operators, some arithmetic operations between positive finitely generated sets must be previously introduced. All these operations would be made $\alpha^{s g}$-point to $\alpha^{s g}$-point.

Definition 17 Let $A, B \in F G([0,1])$. Addition and multiplication between positive finitely generated sets, which will be denoted by + and $\cdot$ respectively, are defined in the following way:

$$
\begin{gathered}
A+B=\bigcup_{\alpha^{s g} \in[0,1]^{\{-,+\}}}\left(K_{\alpha^{s g}}(A)+K_{\alpha^{s g}}(B)\right) . \\
A \cdot B=\bigcup_{\alpha^{s g} \in[0,1]^{\{-,+\}}}\left(K_{\alpha^{s g}}(A) \cdot K_{\alpha^{s g}}(B)\right) .
\end{gathered}
$$

In the previous definition, we have considered the same notation for the addition of two finitely generated sets, the sum of two real numbers and the symbol of one direction. This is an abuse of notation, but we think it is not necessary to consider different symbols, which could difficult the understood of the results, since it is clear the meaning of any of them, depending on the context.

Definition 18 Multiplication by positive scalars can be seen as a multiplication of a finitely generated set $A$ by a degenerated finitely generated set (a scalar) $k \geq 0$, i.e.:

$$
k \cdot A=\bigcup_{\alpha^{s g} \in[0,1]^{\{-,+\}}}\left(K_{\alpha^{s g}}(k) \cdot K_{\alpha^{s g}}(A)\right)=\bigcup_{\alpha^{s g} \in[0,1]^{\{-,+\}}}\left(k \cdot K_{\alpha^{s g}}(A)\right) .
$$

When this multiplication by scalars is extended to $\mathbb{R}$, we have that if $k<0$ then:

$$
k \cdot K_{\alpha^{s g}}(A)=k \cdot K_{(1-\alpha)^{s g^{c}}}(A) \quad \forall \alpha^{s g} \in[0,1]^{\{-,+\}} .
$$

Furthermore, after introducing addition and product, subtraction is the next arithmetic operation to be introduced.

Definition 19 Let $A, B \in F G([0,1])$. Subtraction between positive finitely generated sets is defined in the following way:

$$
A-B=A+(-1) \cdot B \text {. }
$$


Finally, multiplicative inverse and division should be introduced:

Definition 20 Let $A, B \in F G([0,1])$. Multiplicative inverse and division between positive finitely generated sets, which will be denoted by ${ }^{-1}$ and / respectively, are defined in the following way:

$$
\begin{gathered}
B^{-1}=\bigcup_{\alpha^{s g} \in[0,1]^{\{-,+\}}}\left(1 / K_{(1-\alpha)^{s g^{c}}}(B)\right), \\
A / B=A \cdot B^{-1} .
\end{gathered}
$$

Remark 21 It must be remarked that / is not defined when $0 \in B$, since in that case $K_{(1-\alpha)^{s g^{c}}}(B)$ is equal to 0 for some value of $\alpha^{s g} \in[0,1]^{\{-,+\}}$.

Remark 22 The arithmetic operations + and - can be immediately extended to $A, B \in F G([a, b])$ with $a, b \in \mathbb{R}$. However, the same extension of · and / does not keep the properties that we are going to introduce right after when $a<0$.

These operations extended to $F G([a, b])$ with $a, b \in \mathbb{R}$ are going to be used in the following sections when defining aggregation operators. However, this does not constitute a problem as we are only changing the domain of definition.

Remark 23 These operations are not internal in $F G([0,1])$, but they remain being finitely generated sets. It does not suppose any issue.

Next proposition states some properties of these recently defined operations.

Proposition 24 Let $F G([0,1])$ be the class of finitely generated sets in the interval $[0,1]$. Then, the following properties are satisfied:

- Addition and multiplication are commutative.

- Addition and multiplication are associative.

- Multiplication distributes over addition.

- $[0,0]$ and $[1,1]$ are, respectively, identity elements of addition and multiplication.

Proof. It is straightforward as every $\alpha^{s g}$-point is a real number and both + and $\cdot$ are defined from their respective $\alpha^{s g}$-points.

By definition, the following property is straightforward.

Proposition 25 Let $A \in F G([0,1])$. Then, for any $\alpha^{s g} \in[0,1]^{\{-,+\}}$, the following statements are true:

- $K_{\alpha^{s g}}(-A)=-K_{(1-\alpha)^{s g^{c}}}(A)$.

- $K_{\alpha^{s g}}\left(A^{-1}\right)=\left(K_{(1-\alpha)^{s g^{c}}}(A)\right)^{-1}$, if $0 \notin A$. 
An interesting question may arise at this moment. Are the $\alpha^{s g}$-points preserved when working with these operations? The answer is not positive, as we can see in the following counterexample.

Example 26 Let $A, B \in F G([0,1])$ defined by $A=\left[0, \frac{1}{3}\right] \cup\left[\frac{2}{3}, 1\right]$ and $B=$ $\left[\frac{1}{6}, \frac{1}{3}\right] \cup\left[\frac{1}{2}, \frac{2}{3}\right] \cup\left[\frac{5}{6}, 1\right]$. Then,

$A+B=\left[\frac{1}{6}, \frac{5}{9}\right] \cup\left[\frac{13}{18}, \frac{11}{12}\right] \cup\left[\frac{5}{4}, \frac{13}{9}\right] \cup\left[\frac{29}{18}, 2\right]$,

$A-B=\left[-1,-\frac{11}{18}\right] \cup\left[-\frac{4}{9},-\frac{1}{4}\right] \cup\left[\frac{1}{12}, \frac{5}{18}\right] \cup\left[\frac{4}{9}, \frac{5}{6}\right]$,

$A \cdot B=\left[0, \frac{2}{27}\right] \cup\left[\frac{1}{9}, \frac{7}{36}\right] \cup\left[\frac{7}{18}, \frac{14}{27}\right] \cup\left[\frac{35}{54}, 1\right]$ and

$A / B=\left[0, \frac{4}{15}\right] \cup\left[\frac{1}{3}, \frac{7}{12}\right] \cup\left[\frac{7}{6}, \frac{14}{9}\right] \cup\left[\frac{7}{3}, 6\right]$.

However, using $\alpha^{s g}=\frac{1}{3}^{-}$, we have that

$K_{\alpha^{s g}}(A)+K_{\alpha^{s g}}(B)=\frac{2}{9}+\frac{1}{3}=\frac{5}{9} \neq \frac{85}{108}=K_{\alpha^{s g}}(A+B)$,

$K_{\alpha^{s g}}(A)-K_{\alpha^{s g}}(B)=\frac{2}{9}-\frac{1}{3}=-\frac{1}{9} \neq-\frac{41}{108}=K_{\alpha^{s g}}(A-B)$,

$K_{\alpha^{s g}}(A) \cdot K_{\alpha^{s g}}(B)=\frac{2}{9} \cdot \frac{1}{3}=\frac{2}{27} \neq \frac{5}{36}=K_{\alpha^{s g}}(A \cdot B)$,

$K_{\alpha^{s g}}(A) / K_{\alpha^{s g}}(B)=\frac{2}{9} / \frac{1}{3}=\frac{2}{3} \neq \frac{5}{12}=K_{\alpha^{s g}}(A / B)$.

Thus, it is clear that the following statements are not true in general:

- $K_{\alpha^{s g}}(A)+K_{\alpha^{s g}}(B)=K_{\alpha^{s g}}(A+B) \quad \forall \alpha^{s g} \in[0,1]^{\{-,+\}}$.

- $K_{\alpha^{s g}}(A)-K_{(1-\alpha)^{s g^{c}}}(B)=K_{\alpha^{s g}}(A-B) \quad \forall \alpha^{s g} \in[0,1]^{\{-,+\}}$.

- $K_{\alpha^{s g}}(A) \cdot K_{\alpha^{s g}}(B)=K_{\alpha^{s g}}(A \cdot B) \quad \forall \alpha^{s g} \in[0,1]^{\{-,+\}}$.

- $K_{\alpha^{s g}}(A) / K_{(1-\alpha)^{s g^{c}}}(B)=K_{\alpha^{s g}}(A / B) \quad \forall \alpha^{s g} \in[0,1]^{\{-,+\}}$.

We have seen that the $\alpha^{s g}$-points are not preserved when working with these four basic arithmetic operations between finitely generated sets. However, the behaviour of $\alpha^{s g}$-points is not catastrophic and we can define an automorphism $\Psi$ to recover the $\alpha^{s g}$-point only depending of the number of intervals of both finitely generated sets.

Theorem 27 Let $A, B \in F G([0,1])$. Then, there exists a unique increasing injective function $\Psi_{n_{A}, n_{B}}: G\left(n_{A}, n_{B}\right) \rightarrow G\left(n_{A}+n_{B}-\right.$ g.c.d. $\left.\left(n_{A}, n_{B}\right)\right)$ satisfying for any $\alpha^{s g} \in G\left(n_{A}, n_{B}\right)$ that

$$
K_{\alpha^{s g}}(A)+K_{\alpha^{s g}}(B)=K_{\Psi_{n_{A}, n_{B}}\left(\alpha^{s g}\right)}(A+B) .
$$

Proof. Let $A, B \in F G([0,1])$. We define:

$\Psi_{n_{A}, n_{B}}\left(0^{+}\right)=0^{+}$, 
$\Psi_{n_{A}, n_{B}}\left(1^{-}\right)=1^{-}$and

$\Psi_{n_{A}, n_{B}}\left(\alpha_{[i]}^{s g}\right)=\frac{i}{n_{A}+n_{B}-\text { g.c.d. }\left(n_{A}, n_{B}\right)} s g \quad \forall i=1, \ldots, n_{A}+n_{B}-$ g.c.d. $\left(n_{A}, n_{B}\right)-1$,

where $\alpha_{[i]}^{s g}$ is the $i$ th lowest value of $\alpha^{s g}$ among all the $\alpha^{s g} \in G\left(n_{A}, n_{B}\right) \backslash\{0,1\}$.

It is straightforward to see that $\Psi$ is increasing and injective. Let us proof that

$K_{\alpha^{s g}}(A)+K_{\alpha^{s g}}(B)=K_{\Psi_{n_{A}, n_{B}}\left(\alpha^{s g}\right)}(A+B)$.

Let $\alpha^{s g} \in G\left(n_{A}, n_{B}\right) \backslash\{0,1\}$. As $\alpha^{s g} \in G\left(n_{A}, n_{B}\right)$, at least one of $K_{\alpha^{s g}}(A)$ and $K_{\alpha^{s g}}(B)$ is an end (left end if $s g=-$ or right end if $s g=+$ ) of the intervals forming $A$ and $B$ respectively. This means that there is a discontinuity between $K_{\alpha^{-}}(A)+K_{\alpha^{-}}(B)$ and $K_{\alpha^{+}}(A)+K_{\alpha^{+}}(B)$.

As $\alpha^{s g}$ is a generic element of $G\left(n_{A}, n_{B}\right) \backslash\{0,1\}$, we can observe that we have a number of discontinuities equal to a half of the number of elements in $G\left(n_{A}, n_{B}\right) \backslash\{0,1\}$. Due to the continuity in $[0,1]^{s g} \backslash G\left(n_{A}, n_{B}\right)$ and the increasing monotonicity of the operator "+" with respect to its two components, it follows that the number of discontinuities plus 1 is the number of intervals in $A+B$ and that every end of interval in $A+B$ is obtained as the addition of $K_{\alpha^{s g}}(A)$ and $K_{\alpha^{s g}}(B)$ with $\alpha^{s g} \in G\left(n_{A}, n_{B}\right)$.

Finally, let us consider $\alpha_{[i]}^{s g} \in G\left(n_{A}, n_{B}\right) \backslash\{0,1\}$ the $i$-th lowest value of $\alpha^{s g}$ among all the $\alpha^{s g} \in G\left(n_{A}, n_{B}\right) \backslash\{0,1\}$. We can see that $K_{\alpha^{s g}}(A+B)$ may not coincide with the corresponding to $K_{\alpha^{s g}}(A)$ and $K_{\alpha^{s g}}(B)$ because $n_{A+B}$ may not coincide with $n_{A}$ and $n_{B}$. This point is associated to $\frac{i}{n_{A}+n_{B}-\text { g.c.d. }\left(n_{A}, n_{B}\right)} s g$.

On the other hand, uniqueness is straightforward due to the increasing monotonicity of $\Psi$ and of the $\alpha^{s g}$-point operator, i.e. we are looking for a bijection between $G\left(n_{A}, n_{B}\right)$ and $G\left(n_{A}+n_{B}-\right.$ g.c.d. $\left.\left(n_{A}, n_{B}\right)\right)$ and as there is a linear order between the elements in both sets and the function needs to be increasing, we have $\Psi$ as the unique possibility. Therefore, $\Psi$ is the unique increasing one-to-one function between $G\left(n_{A}, n_{B}\right)$ and $G\left(n_{A}+n_{B}-\right.$ g.c.d. $\left.\left(n_{A}, n_{B}\right)\right)$.

We have just stated that there exists a unique increasing function relating the grid points of two sets and the result of operating them. In the following proposition an extension to $[0,1]^{\{-,+\}}$of this function $\Psi$ is proposed.

Proposition 28 This function $\Psi_{n_{A}, n_{B}}$ can be extended to $[0,1]^{\text {sg }}$ satisfying

$K_{\alpha^{s g}}(A)+K_{\alpha^{s g}}(B)=K_{\Psi_{n_{A}, n_{B}}\left(\alpha^{s g}\right)}(A+B)$,

$\forall \alpha^{s g} \in[0,1]^{s g} \quad \forall A, B \in F G([0,1])$.

Proof. Let $\Psi$ be the function introduced in Theorem 27 . We would like to obtain $\Psi_{n_{A}, n_{B}}:[0,1]^{\{-,+\}} \backslash G\left(n_{A}, n_{B}\right) \rightarrow[0,1]^{\{-,+\}}$ 
Let $i$ be the natural number satisfying $\alpha^{s g} \in\left(\beta_{i-1}^{s g}, \beta_{i}^{s g}\right)$, with $\beta_{i-1}^{s g}, \beta_{i}^{s g} \in$ $G\left(n_{A}, n_{B}\right)$ two consecutive elements of the $n_{A}, n_{B}$-grid. Then,

$\Psi_{n_{A}, n_{B}}\left(\alpha^{s g}\right)=\Psi_{n_{A}, n_{B}}\left(\beta_{i-1}^{s g}\right)+\alpha^{\prime}\left(\Psi_{n_{A}, n_{B}}\left(\beta_{i}^{s g}\right)-\Psi_{n_{A}, n_{B}}\left(\beta_{i-1}^{s g}\right)\right)$,

where $\alpha^{\prime}=\frac{\alpha-\beta_{i-1}}{\beta_{i}-\beta_{i-1}}$.

The proof of $K_{\alpha^{s g}}(A)+K_{\alpha^{s g}}(B)=K_{\Psi_{n_{A}, n_{B}}\left(\alpha^{s g}\right)}(A+B)$ is straightforward considering Proposition 7 with $\beta_{i-1}, \alpha$ and $\beta_{i}$ and assuming Theorem 27.

Corollary $29 \Psi_{n_{A}, n_{B}}$ extended to $[0,1]^{s g}$ is an increasing bijective function. Furthermore, $\Psi_{n_{A}, n_{B}}:[0,1]^{s g} \rightarrow[0,1]^{\text {sg }}$ is an automorphism.

Finally, we conclude under which conditions the $\alpha^{s g}$-points are preserved in the following theorem.

Theorem 30 Let $A, B \in F G([0,1])$. Then, the following statements are equivalent:

(1) $n_{A} \mid n_{B}$ or $n_{B} \mid n_{A}$.

(2) $\Psi_{n_{A}, n_{B}}=I d$.

(3a) $K_{\alpha^{s g}}(A)+K_{\alpha^{s g}}(B)=K_{\alpha^{s g}}(A+B) \quad \forall \alpha^{s g} \in[0,1]^{\{-,+\}}$.

(3b) $K_{\alpha^{s g}}(A)+K_{\alpha^{s g}}(B)=K_{\alpha^{s g}}(A+B) \quad \forall G\left(n_{A}, n_{B}\right)$.

(4a) $K_{\alpha^{s g}}(A)-K_{(1-\alpha)^{s g^{c}}}(B)=K_{\alpha^{s g}}(A-B) \quad \forall \alpha^{s g} \in[0,1]^{\{-,+\}}$.

(4b) $K_{\alpha^{s g}}(A)-K_{(1-\alpha)^{s c}}(B)=K_{\alpha^{s g}}(A-B) \quad \forall G\left(n_{A}, n_{B}\right)$.

(5) $K_{\alpha^{s g}}(A) \cdot K_{\alpha^{s g}}(B)=K_{\alpha^{s g}}(A \cdot B) \quad \forall G\left(n_{A}, n_{B}\right)$.

(6) $K_{\alpha^{s g}}(A) / K_{(1-\alpha)^{s g^{c}}}(B)=K_{\alpha^{s g}}(A / B) \quad \forall G\left(n_{A}, n_{B}\right)$.

Proof. $\quad(1) \Rightarrow(2)$ : Let us consider, without loss of generality, $n_{A} \mid n_{B}$. Then, g.c.d. $\left(n_{A}, n_{B}\right)=n_{A}$ and therefore $G\left(n_{A}, n_{B}\right)=G\left(n_{B}\right)$, i.e. every element of $G\left(n_{A}, n_{B}\right)$ can be represented as ${\frac{1}{n_{B}}}^{s g}$.

Let $\alpha_{[i]}^{s g}$ be the $i$ th lowest value of $\alpha^{s g}$ among all the $\alpha^{s g} \in G\left(n_{A}, n_{B}\right) \backslash\{0,1\}$.

$\Psi_{n_{A}, n_{B}}\left(\alpha_{[i]}^{s g}\right)={\frac{i}{n_{A}+n_{B}-\text { g.c.d. }\left(n_{A}, n_{B}\right)}}^{s g}={\frac{i}{n_{B}}}^{s g}=\alpha_{[i]}^{s g}$.

$(2) \Rightarrow(3 a)$ : By Theorem 27 we have

$K_{\alpha^{s g}}(A)+K_{\alpha^{s g}}(B)=K_{\Psi_{n_{A}, n_{B}}\left(\alpha^{s g}\right)}(A+B) \quad \forall \alpha^{s g} \in G\left(n_{A}, n_{B}\right)$.

Finally, we can use Proposition 28 to extend the previous result to $[0,1]^{s g}$ and apply that $\Psi_{n_{A}, n_{B}}=I d$ to obtain

$K_{\alpha^{s g}}(A)+K_{\alpha^{s g}}(B)=K_{\alpha^{s g}}(A+B) \quad \forall \alpha^{s g} \in[0,1]^{\{-,+\}}$.

$(3 a) \Rightarrow(3 b)$ : Direct. 
$(3 b) \Rightarrow(4 b)$ : If $K_{\alpha^{s g}}(A)+K_{\alpha^{s g}}(B)=K_{\alpha^{s g}}(A+B) \quad \forall G\left(n_{A}, n_{B}\right)$ then a correction $\Psi$ of the $\alpha^{s g}$-points is not needed and, by Theorem 27, $\Psi=I d$. Finally, as $\Psi$ only depends on $n_{A}$ and $n_{B}$ (and $n_{B}=n_{-B}$ ), we have that $K_{\alpha^{s g}}(A)+$ $K_{\alpha^{s g}}(-B)=K_{\alpha^{s g}}(A+(-B)) \quad \forall G\left(n_{A}, n_{B}\right)$, i.e. $K_{\alpha^{s g}}(A)-K_{(1-\alpha)^{s g^{c}}}(B)=$ $K_{\alpha^{s g}}(A-B) \quad \forall G\left(n_{A}, n_{B}\right)$.

$(4 b) \Rightarrow(4 a)$ : Let $\beta_{i-1}^{+}, \beta_{i}^{-} \in G\left(n_{A}, n_{B}\right)$ two consecutive elements of the $n_{A}, n_{B^{-}}$ grid. Let $\alpha^{s g} \in[0,1]^{\{-,+\}}$such that $\beta_{i-1}^{+}<\alpha^{s g}<\beta_{i}^{-}$. We can apply Proposition 7 to $A,-B$ and $A+(-B)$ and considering (4b) with $\beta_{i-1}^{+}$and $\beta_{i}^{-}$, we obtain that:

$K_{\alpha^{s g}}(A)-K_{(1-\alpha)^{s g}}(B)=K_{\alpha^{s g}}(A-B) \quad \forall \alpha^{s g} \in[0,1]^{\{-,+\}}$.

$(4 a) \Rightarrow(5)$ :

First of all we are going to prove that, in this case, $\Psi_{n_{A}, n_{B}}=I d$.

$K_{\alpha^{s g}}(A)-K_{(1-\alpha)^{s g^{c}}}(B)=K_{\alpha^{s g}}(A-B) \quad \forall \alpha^{s g} \in[0,1]^{\{-,+\}}$is equivalent to $K_{\alpha^{s g}}(A)+K_{\alpha^{s g}}(-B)=K_{\alpha^{s g}}(A-B) \quad \forall \alpha^{s g} \in[0,1]^{\{-,+\}}$. Therefore a correc-

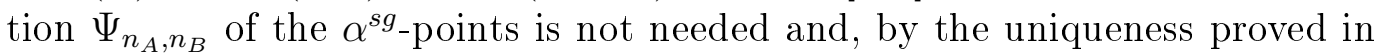
Theorem 27, $\Psi_{n_{A}, n_{B}}=I d$.

On the other hand, an analogous reasoning to the one used in the proof of Theorem 27 may derive on:

$K_{\alpha^{s g}}(A) \cdot K_{\alpha^{s g}}(B)=K_{\Psi_{n_{A}, n_{B}}\left(\alpha^{s g}\right)}(A \cdot B) \quad \forall \alpha^{s g} \in G\left(n_{A}, n_{B}\right)$.

Finally, we apply that $\Psi_{n_{A}, n_{B}}=I d$ to obtain

$K_{\alpha^{s g}}(A) \cdot K_{\alpha^{s g}}(B)=K_{\alpha^{s g}}(A \cdot B) \quad \forall G\left(n_{A}, n_{B}\right)$.

$(5) \Rightarrow(6)$ : Direct. It is enough to remind that $A / B=A \cdot B^{-1}$ and $K_{\alpha^{s g}}\left(B^{-1}\right)=$ $\left(K_{(1-\alpha)^{s g^{c}}}(B)\right)^{-1}$.

$(6) \Rightarrow(1): K_{\alpha^{s g}}(A) / K_{(1-\alpha)^{s g^{c}}}(B)=K_{\alpha^{s g}}(A / B) \quad \forall G\left(n_{A}, n_{B}\right)$ is equivalent to $K_{\alpha^{s g}}(A) \cdot K_{\alpha^{s g}}\left(B^{-1}\right)=K_{\alpha^{s g}}(A / B) \quad \forall G\left(n_{A}, n_{B}\right)$. Therefore, repeating the analogous reasoning of $(4 a) \Rightarrow(5)$, the function $\Psi_{n_{A}, n_{B}}$ needs to be the identity.

On the other hand, if $\Psi_{n_{A}, n_{B}}=I d$, then $\alpha_{[i]}^{s g}={\frac{i}{n_{A}+n_{B}-\text { g.c.d. }\left(n_{A}, n_{B}\right)}}^{s g} \forall i=$ $1, \ldots, n_{A}+n_{B}-$ g.c.d. $\left(n_{A}, n_{B}\right)-1$,

where $\alpha_{[i]}^{s g}$ is the $i$ th lowest value of $\alpha^{s g}$ among all the $\alpha^{s g} \in G\left(n_{A}, n_{B}\right) \backslash\{0,1\}$.

This means that $G\left(n_{A}, n_{B}\right)=G\left(n_{A}+n_{B}-\right.$ g.c.d. $\left.\left(n_{A}, n_{B}\right)\right)$. Finally, by Proposition 12, a grid generated by two elements is only equal to a grid generated by one element if there is a divisor relation between the two elements generating 
the first grid. Therefore, $n_{A} \mid n_{B}$ or $n_{B} \mid n_{A}$.

Remark 31 It must be remarked that, besides it is true for elements in $G\left(n_{A}, n_{B}\right)$, product and division do not fulfil Theorem 30 for elements in $[0,1]^{s g} \backslash G\left(n_{A}, n_{B}\right)$, as it proves the following example.

Example 32 Let $A=[0.1,0.2]$ and $B=[0.8,1]$. Therefore, we can see that $n_{A}=1=n_{B}\left(n_{A} \mid n_{B}\right.$ and $\left.n_{B} \mid n_{A}\right)$.

However we can see that for $\alpha^{s g}=0.5^{+} \notin G\left(n_{A}, n_{B}\right)$,

$K_{\alpha^{s g}}(A) \cdot K_{\alpha^{s g}}(B) \neq K_{\alpha^{s g}}(A \cdot B)$,

$K_{\alpha^{s g}}(A) / K_{(1-\alpha)^{s g^{c}}}(B) \neq K_{\alpha^{s g}}(A / B)$.

$K_{0.5^{+}}(A)=0.15$ and $K_{0.5^{+}}(B)=K_{0.5^{+}}(B)=0.9$

$A \cdot B=[0.08,0.2]$ and $A / B=[0.1,0.25]$.

$K_{0.5^{+}}(A \cdot B)=0.14 \neq 0.135=0.15 \cdot 0.9=K_{0.5^{+}}(A) \cdot K_{0.5^{+}}(A)$,

$K_{0.5^{+}}(A / B)=0.175 \neq 0.16667=0.15 / 0.9=K_{0.5^{+}}(A) / K_{0.5^{-}}(A)$.

\subsection{Aggregation operators}

Aggregation of several input values into a single output value is an indispensable tool in several branches of Applied Mathematics, especially in group decision making. A good review about aggregation operators can be found in [4]. In the usual case, the domain of the operator is based on a closed interval of the real line. However, in our context, we need to extend this definition for the case where we are dealing with finitely generated sets. Aggregation operators between finitely generated sets can be defined as follows:

Definition 33 An n-ary aggregation operator (or n-ary aggregation function) for finitely generated sets is a function Agg ${ }^{(n)}$ which assigns a finitely generated set to any $n$ finitely generated sets $x_{1}, x_{2}, \ldots, x_{n}$, that $i s$,

$$
\begin{aligned}
\operatorname{Agg}^{(n)}: \quad F G([0,1])^{n} & \longrightarrow \quad F G([0,1]) \\
\left(x_{1}, x_{2}, \ldots, x_{n}\right) & \longrightarrow \operatorname{Agg}^{(n)}\left(x_{1}, x_{2}, \ldots, x_{n}\right)
\end{aligned},
$$

such that it fulfils two conditions:

- Increasing: if $x_{i} \leq_{l o} y_{i}$ for each $i \in\{1,2, \ldots, n\}$, then:

$$
\operatorname{Agg}^{(n)}\left(x_{1}, x_{2}, \ldots, x_{n}\right) \leq_{l o} \operatorname{Agg}^{(n)}\left(y_{1}, y_{2}, \ldots, y_{n}\right) .
$$


- Boundary conditions:

$$
\inf _{x \in F G([0,1])^{n}} A g g^{(n)}(x)=[0,0] \text { and } \sup _{x \in F G([0,1])} A g g^{(n)}(x)=[1,1] .
$$

Definition 34 An aggregation operator (or extended aggregation function) for finitely generated sets is a function Agg : $\bigcup_{n \in \mathbb{N}} F G([0,1])^{n} \longrightarrow F G([0,1])$ such that for all $n>1,\left.A g g^{(n)}\right|_{F G([0,1])^{n}}$ is an n-ary aggregation function and $A g g^{(1)}$ is the identity on $[0,1]$.

Despite more aggregation operators could be defined, introducing arithmetic mean is enough in order to analyse the aim of this paper: a twofold group decision making problem. This will be the aggregation operator considered in the case study of Section 5 .

Definition 35 Let $n \in \mathbb{N}$ and $x=\left(x_{1}, \ldots, x_{n}\right) \in F G([0,1])^{n}$. Therefore, the aggregation operator $A M$, defined via

$$
A M(x)=\left(\sum_{i=1}^{n} x_{i}\right) / n
$$

is called arithmetic mean.

Proposition $36 A M$ is an aggregation operator.

\section{Proof.}

Let $n \in \mathbb{N}$ and $x=\left(x_{1}, \ldots, x_{n}\right) \in F G([0,1])^{n}$. Therefore,

$$
A M(x)=\bigcup_{\alpha^{s g} \in[0,1]^{\{-,+\}}}\left(\sum_{i=1}^{n} K_{\alpha^{s g}}\left(x_{i}\right)\right) / n .
$$

As the sum of finitely generated sets is still a finitely generated set and we can divide by scalars and remain in FGs, we have that $A M(x)$ is still a FG. Let us check if increasing and boundary conditions are satisfied.

\section{Increasing:}

Let $y=\left(y_{1}, \ldots, y_{n}\right) \in F G([0,1])^{n}$ such that $x_{i} \leq_{l o} y_{i} \forall i \in\{1,2, \ldots, n\}$. By definition of $\leq_{l o}, K_{\alpha^{s g}}\left(x_{i}\right) \leq K_{\alpha^{s g}}\left(y_{i}\right), \forall \alpha^{s g} \in[0,1]^{\{-,+\}}$and $\forall i \in\{1,2, \ldots, n\}$.

Therefore,

$$
A M(x)=\bigcup_{\alpha^{s g} \in[0,1]^{\{-,+\}}}\left(\sum_{i=1}^{n} K_{\alpha^{s g}}\left(x_{i}\right)\right) / n \leq \bigcup_{\alpha^{s g} \in[0,1]^{\{-,+\}}}\left(\sum_{i=1}^{n} K_{\alpha^{s g}}\left(y_{i}\right)\right) / n=A M(y) .
$$

\section{Boundary conditions:}


Let $z=[0,0]^{n}$. Hence, as $\inf (F G([0,1]))=[0,0], A M(z)=[0,0]$ and $A M$ is increasing, we have that

$$
\inf _{x \in F G([0,1])^{n}} A M(x)=[0,0] .
$$

Analogously, let $z=[1,1]^{n}$. Hence, as $\sup (F G([0,1]))=[1,1], A M(z)=[1,1]$ and $A M$ is proved increasing, we have that

$$
\sup _{x \in F G([0,1])^{n}} A M(x)=[1,1] .
$$

\section{Twofold group decision making}

In a classical group decision making problem, we have a set of $n_{a}$ alternatives $X=\left\{x_{1}, \ldots, x_{n_{a}}\right\},\left(n_{a} \geq 2\right)$ and a set of $n$ experts $E=\left\{e_{1}, \ldots, e_{n}\right\},(n \geq 2)$. Each expert provides his preferences on the set of alternatives and the goal of the group decision making problem is to look for the alternative (or set of alternatives) which is (are) most accepted by the experts.

The resolution of a classical group decision making problem, according with the principles established in [6], is developed in the following two steps:

(1) Making the information uniform. Each preference ordering of each expert is transformed into a fuzzy preference relation form.

(2) Application of a selection process. The most accepted alternative by our experts must be selected.

In addition, the selection process is also applied in two steps:

(2a) Aggregation phase. A consensus fuzzy preference relation is obtained using an aggregation operator.

(2b) Exploitation phase. The most accepted alternative from the consensus fuzzy preference relation is selected.

However, the aim of this paper is to improve this classical group decision making problem, generalizing it using finite interval-valued hesitant fuzzy preference relations. This improvement allows us to consider at the same time several criteria and experts and to model the uncertainty derived from the inaccurate information we are working with.

In the twofold case, we have a set of $n_{a}$ alternatives $X=\left\{x_{1}, \ldots, x_{n_{a}}\right\},\left(n_{a} \geq\right.$ $2)$, a set of $n$ criteria $C=\left\{c_{1}, \ldots, c_{n}\right\},(n \geq 2)$ and a set of $n^{c_{i}}$ experts in each criteria $E^{i}=\left\{e_{1}^{i}, \ldots, e_{n^{c_{i}}}^{i}\right\}, \quad\left(n^{c_{i}} \geq 1\right), \forall i \in\{1, \ldots, n\}$.

The goal of the twofold group decision making problem is to look for the 
alternative (or set of alternatives) which is (are) most accepted by the experts, according to the different criteria. The steps to model these problems are the following:

(1) Making the information uniform. Each preference ordering of each criteria is transformed into a finite interval-valued hesitant fuzzy preference relation form.

(1a) Joint phase. A consensus between the experts in each criteria is hold.

(1b) Preference phase. Joined information is transformed into a finite interval-valued hesitant fuzzy preference relation form.

(2) Application of a selection process. The most accepted alternative by our experts must be selected.

(2a) Aggregation phase. A consensus finite interval-valued hesitant fuzzy preference relation is obtained using an aggregation operator.

(2b) Exploitation phase. The most accepted alternative from the consensus finite interval-valued hesitant fuzzy preference relation is selected.

Let us deeply analysed each phase in the following subsections.

\subsection{Making the information uniform: Joint phase}

In the twofold problem we will initially have some information provided by several experts in different criteria. Then, we will construct a finite interval-valued hesitant fuzzy preference relation for each criteria joining the considerations of the experts in the same criteria. As we know, a finite interval-valued hesitant fuzzy preference relation is given (when $n_{a}$ alternatives) in the form of an $n_{a} \times n_{a}$ matrix where the element $p_{i j}^{k}$ of the $i$-th row and $j$-th column is a finitely generated set representing the finite interval-valued hesitant fuzzy preference of the alternative $x_{i}$ over the alternative $x_{j}$ according to the criteria $c_{k}$. However, this step is the most flexible one due to the nature of the initial data, where our information could be expressed in several ways.

The most common way in which each expert gives information is as an interval of utility values. Note that the smallest the length of the interval is the most accurate is the given information. As it was introduced, the goal of this subsection would be to join the information of all the experts in the same criteria.

Therefore, the joint of the information is made using the $\cup$ operator in order to obtain a finitely generated set formed by the union of every interval utility value given by the experts.

Let $v_{i, j}^{k}$ be the interval utility value provided by the $j$-th expert in the criteria $c_{k}$ to the $i$-th object. 
Then, $v_{i}^{k}=\bigcup_{i=1}^{n^{c_{k}}} v_{i, j}^{k}$ would be the finitely generated set utility value provided by the experts in the criteria $c_{k}$ to the $i$-th object.

\subsection{Making the information uniform: Preference phase}

Once we have joint the information given by the experts in the same criteria, $n$ finite interval-valued hesitant fuzzy preference relations (one corresponding to each criteria) are going to be constructed. This is going to be done using a fuzzy preference relation.

In [19], Pérez et al. proved that a parametrically continuous fuzzy preference relation could generate a finite interval-valued hesitant fuzzy preference relation under certain boundary conditions. In our case, these boundary conditions are reduced to have a finite number of experts in each criteria. This generated finite interval-valued hesitant fuzzy preference relation $\sigma(R)$, when defined over finitely generated set, is characterized as follows:

$$
\sigma(R)(A, B)=\bigcup_{\alpha^{s g}} R\left(K_{\alpha^{s g}}(A), K_{\alpha^{s g}}(B)\right)
$$

Therefore, choosing a parametrically continuous fuzzy preference relation $R$, $\sigma(R)$ will be used to construct $n$ finite interval-valued hesitant preference relations $P^{i}$ (where $n$ is the number of criteria) using the interval utility values obtained in the joint phase. Reader may refer to $[7,16,28]$ to obtain some examples of fuzzy preference relations.

Once the information has been made uniform, the real initial point of a group decision making problem has been achieved and the selection process can be tackled.

\subsection{Application of a selection process: Aggregation phase}

In this phase our goal is to select the right aggregation operator. Once we have fixed the aggregation operator $A$ that we are going to consider, this phase is reduced to apply this aggregation operator in order to obtain a consensus finite interval-valued hesitant fuzzy preference relation, defined via:

$$
p_{i j}=A\left(p_{i j}^{1}, \ldots, p_{i j}^{n}\right) \quad \forall i, j=1, \ldots, n_{a},
$$

where $P^{k}$ is the finite interval-valued hesitant fuzzy preference relation according to the $k$ th criterion, and $p_{i j}^{k}$ is the element in the $i$-th row and $j$-th column of the matrix $P^{k}$. 


\subsection{Application of a selection process: Exploitation phase}

Finally, we have a finite interval-valued hesitant fuzzy preference relation in its matrix form. We are going to use the Extended Weighted Voting Method introduced in [18]. The purpose of this extended method is to use a parameter $\alpha$ allowing us to model the importance of "being desirable" over the other alternatives and of "not being preferred" by the other alternatives. However, this method must be retouched in order to operate with finite interval-valued hesitant fuzzy preference relations.

\section{Algorithm 1 Twofold Extended Weighted Voting Method (T-EWVM)}

\section{- Input:}

- A finite interval-valued hesitant fuzzy preference relation $P$ over a set of alternatives $\mathcal{A}=\left\{a_{1}, \ldots, a_{n}\right\}$

- Fix the parameter $\alpha$

- Choose an aggregation operator A between finitely generated sets

- Choose an order $\leq_{*}$ between finitely generated sets

- Output: A family of non-empty sets $\mathcal{K}_{i}$

1. Normalize (if not) our finite interval-valued hesitant fuzzy preference relation

2. Separate positive preference $\left(P^{+}\right)$and negative preference $\left(P^{-}\right)$via

$$
\begin{gathered}
p_{i j}^{+}=\left\{\begin{array}{cc}
\emptyset & \text { if } \max \left(p_{i j}\right)<0.5 \\
I_{p_{i j} \geq 0.5}-[0.5,0.5] & \text { if } \max \left(p_{i j}\right) \geq 0.5
\end{array}\right. \\
p_{i j}^{+}= \begin{cases}\emptyset & \text { if } \max \left(p_{i j}\right)<0.5 \\
I_{p_{i j} \geq 0.5}-[0.5,0.5] & \text { if } \max \left(p_{i j}\right) \geq 0.5\end{cases}
\end{gathered}
$$

where $I_{A R x}=\bigcup_{\alpha^{s g} \in[0,1]^{\{-,+\}}}\left\{K_{\alpha^{s g}}(A) \mid K_{\alpha^{s g}}(A) R x\right\}$

with $A \in F G([0,1]), R \in\{<, \leq,>, \geq\}$ and $x \in \mathbb{R}$.

3. $P^{\alpha}=\alpha \cdot P^{+} \cup(1-\alpha) \cdot P^{-}$

4. $\mathcal{B}_{0}=\mathcal{A}$ and $k=0$

5. While $\mathcal{B}_{k} \neq \emptyset$

For each $a_{i} \in \mathcal{B}_{k}$

$$
\begin{aligned}
& \mathbb{M}_{i}=\left\{l \in\{1, \ldots, n\} / a_{l} \in \mathcal{B}_{k} \text { and } l \neq i\right\} \\
& I\left(a_{i}\right)=\operatorname{Agg}\left(p_{i \mathbb{M}_{i}}^{\alpha}\right)
\end{aligned}
$$

$\mathcal{K}_{k+1}=a_{\operatorname{argmax}_{i}\left\{I\left(a_{i}\right)\right\}}$ (w.r.t. $\left.\leq_{*}\right)$

$\mathcal{B}_{k+1}=\mathcal{B}_{k} \backslash \mathcal{K}_{k+1}$

$k=k+1$

end

Proposition 37 This algorithm reduced to fuzzy preference relations returns 
the original EWVM seen in [18].

Proof. There are only two differences between Algorithm 1 and the algorithm seen in [18].

The first one is the way we define $P^{+}$and $P^{-}$. Note that, if reduced to singletons, $p_{i j}^{+}=\emptyset$ or $p_{i j}^{-}=\emptyset$ or $p_{i j}^{+}=p_{i j}^{-}=0$. Thus, the value of $p_{i j}^{\alpha}$ (calculated as the union of $\alpha p_{i j}^{+}$and $\left.(1-\alpha) p_{i j}^{-}\right)$is a singleton.

On the other hand, the original algorithm would return $p_{i j}^{+}=0$ or $p_{i j}^{-}=0$ or $p_{i j}^{+}=p_{i j}^{-}=0$. Thus, the value of $p_{i j}^{\alpha}$ (calculated as the sum of $\alpha p_{i j}^{+}$and $\left.(1-\alpha) p_{i j}^{-}\right)$coincides with the value obtained with the new algorithm.

The second difference is that in the finite interval-valued hesitant fuzzy version of the algorithm indexes $I\left(a_{i}\right)$ are finitely generated sets. However, when reduced to singletons, every order between finitely generated sets $\leq_{*}$ respects the usual order between real numbers.

In [18], it is shown how a total order can be constructed using the family of non-empty sets $\mathcal{K}_{i}$ that Algorithm 1 outputs.

Remark 38 The behaviour of this method depending on the parameter $\alpha \in$ $[0,1]$ is the same for finite interval-valued hesitant fuzzy preference relations that the one we had for fuzzy preference relations ([18]):

If $\alpha=0.5$, we have exactly the weighted voting method.

If $\alpha<0.5$, we give preference to the least dominated alternative.

If $\alpha>0.5$, we give preference to the most dominating alternative.

\section{Case study}

The aim of this section is to illustrate the right use of a resolution of a twofold group decision making problem with an easy practical example. Let us analyse a problem where we want to study the desirability of 4 objects $\left(O_{1}, O_{2}, O_{3}\right.$ and $\mathrm{O}_{4}$ ) according to the good quality-to-price ratio with the help of 5 experts. Among our 5 experts, 3 are experts in the quality area $\left(e_{1}^{1}, e_{2}^{1}\right.$ and $\left.e_{3}^{1}\right)$ and the other 2 are experienced in the economical area $\left(e_{1}^{2}\right.$ and $\left.e_{2}^{2}\right)$. Each expert has to evaluate each object with an utility value in a $1-10$ scale. As there exists a reasonable quantity of uncertainty, the experts are allowed to use utility intervals instead of utility values.

Let us identify each element of our twofold group decision making problem: 
- The set of $n_{a}=4$ alternatives are the objects to study $X=\left\{O_{1}, O_{2}, O_{3}, O_{4}\right\}$.

- The set of $n=2$ criteria $C=\left\{c_{1}=\right.$ Quality, $c_{2}=$ Economy $\}$.

- The set of $n^{c_{1}}=3$ experts in the first criteria is $E^{1}=\left\{e_{1}^{1}, e_{2}^{1}, e_{3}^{1}\right\}$.

- The set of $n^{c_{2}}=2$ experts in the second criteria is $E^{2}=\left\{e_{1}^{2}, e_{2}^{2}\right\}$.

In Table 1 we can see the results of the analysis made by the experts with its respective evaluations of each object.

Table 1

Analysis of the experts.

\begin{tabular}{|c|c|c|c|c|c|}
\cline { 2 - 6 } \multicolumn{1}{c|}{} & \multicolumn{4}{c|}{ Experts } \\
\cline { 2 - 6 } \multicolumn{1}{c|}{} & \multicolumn{3}{c|}{ Quality } & \multicolumn{2}{c|}{ Economy } \\
\hline$O_{1}$ & 8 & {$[8,9]$} & 10 & {$[1,2]$} & {$[1,3]$} \\
\hline$O_{2}$ & 5 & {$[3,5]$} & {$[7,8]$} & {$[3,4]$} & 5 \\
\hline$O_{3}$ & {$[4,5]$} & {$[3,4]$} & 4 & {$[5,6]$} & {$[5,6]$} \\
\hline$O_{4}$ & {$[9,10]$} & {$[6,7]$} & {$[5,8]$} & {$[7,8]$} & 9 \\
\hline
\end{tabular}

Once we have tested the preliminary data, we must begin with the first step of the study of a twofold group decision making problem: making the information uniform. This part, as we have already said, is the most flexible one due to the nature of the initial data. In our case we have utility intervals and we are looking for a finite interval-valued hesitant fuzzy preference relation for each criteria.

In the Joint phase, the $U$ operator is selected in order to join the information provided by the experts in the same criteria. The obtained results are shown in Table 2. We can see, for example, how to obtain the first element.

$$
v_{1}^{Q}=\bigcup_{i=1}^{n^{Q}} v_{1, i}^{Q}=\{8\} \cup[8,9] \cup\{10\}=[8,9] \cup\{10\} .
$$

In the Preference phase, considering the analysis of Table 2, we can obtain a Table 2

Reduced analysis of the experts.

\begin{tabular}{|c|c|c|}
\cline { 2 - 3 } \multicolumn{1}{c|}{} & \multicolumn{2}{c|}{ Experts } \\
\cline { 2 - 3 } \multicolumn{1}{c|}{} & Quality & Economy \\
\hline$O_{1}$ & {$[8,9] \cup\{10\}$} & {$[1,3]$} \\
\hline$O_{2}$ & {$[3,5] \cup[7,8]$} & {$[3,4] \cup\{5\}$} \\
\hline$O_{3}$ & {$[3,5]$} & {$[5,6]$} \\
\hline$O_{4}$ & {$[5,8] \cup[9,10]$} & {$[7,8] \cup\{9\}$} \\
\hline
\end{tabular}

finite interval-valued hesitant fuzzy preference relation for each criterion using 
a parametrically continuous fuzzy preference relation. In this practical case, we are going to use one of the easiest parametrically continuous fuzzy preference relation which is defined via:

$$
p_{i j}=\frac{u_{i}}{u_{i}+u_{j}} \quad \forall i, j=1, \ldots, n,
$$

where $u_{i}$ and $u_{j}$ are the utility values (supposed singletons) of alternatives $a_{i}$ and $a_{j}$ respectively.

Remember that the generated finite interval-valued hesitant fuzzy preference relation is:

$$
\sigma(p)_{i j}^{k}=\bigcup_{\alpha^{s g} \in[0,1]^{\{-,+\}}} \frac{K_{\alpha^{s g}}\left(v_{i}^{k}\right)}{K_{\alpha^{s g}}\left(v_{i}^{k}\right)+K_{\alpha^{s g}}\left(v_{j}^{k}\right)} \quad \forall i, j=1, \ldots, n,
$$

where $v_{i}^{k}$ and $v_{j}^{k}$ are the utility values (finitely generated sets of Table 2) of objects $O_{i}$ and $O_{j}$ respectively with relation to the criterion $c_{k}$.

$$
\begin{aligned}
& \sigma(p)^{Q}=\left(\begin{array}{cccc}
{[0.500,0.500]} & {[0.556,0.588] \cup[0.643,0.727]} & {[0.667,0.727]} & {[0.500,0.526] \cup[0.529,0.615]} \\
{[0.273,0.357] \cup[0.412,0.444]} & {[0.500,0.500]} & {[0.500,0.556] \cup[0.615,0.636]} & {[0.375,0.385] \cup[0.438,0.444]} \\
{[0.273,0.333]} & {[0.364,0.385] \cup[0.444,0.500]} & {[0.500,0.500]} & {[0.308,0.375]} \\
{[0.385,0.471] \cup[0.474,0.500]} & {[0.556,0.563] \cup[0.615,0.625]} & {[0.625,0.692]} & {[0.500,0.500]}
\end{array}\right) \\
& \sigma(p)^{E}=\left(\begin{array}{ccccc}
{[0.500,0.500]} & {[0.250,0.375]} & {[0.167,0.333]} & {[0.125,0.250]} \\
{[0.625,0.750]} & {[0.500,0.500]} & {[0.375,0.421] \cup[0.455,0.476]} & {[0.300,0.333] \cup[0.357,0.357]} \\
{[0.667,0.833]} & {[0.524,0.546] \cup[0.579,0.625]} & {[0.500,0.500]} & {[0.379,0.400] \cup[0.407,0.417]} \\
{[0.750,0.875]} & {[0.643,0.643] \cup[0.667,0.700]} & {[0.583,0.593] \cup[0.600,0.621]} & {[0.500,0.500]}
\end{array}\right)
\end{aligned}
$$

At this moment we have finished the first step: making the information uniform, and it is time to start with the Selection process, more precisely with the Aggregation phase. First of all, an aggregation operator Agg must be selected. In this paper, in order to do not complicate the process, the arithmetic mean is chosen.

$$
\begin{aligned}
& M=A\left(\sigma(p)^{Q}, \sigma(p)^{E}\right)= \\
& =\left(\begin{array}{cccc}
{[0.500,0.500]} & {[0.403,0.450] \cup[0.478,0.551]} & {[0.417,0.530]} & {[0.313,0.357] \cup[0.359,0.433]} \\
{[0.449,0.522] \cup[0.550,0.597]} & {[0.500,0.500]} & {[0.438,0.488] \cup[0.535,0.556]} & {[0.338,0.359] \cup[0.397,0.401]} \\
{[0.470,0.583]} & {[0.444,0.465] \cup[0.512,0.563]} & {[0.500,0.500]} & {[0.343,0.371] \cup[0.374,0.396]} \\
{[0.567,0.642] \cup[0.643,0.688]} & {[0.599,0.603] \cup[0.641,0.663]} & {[0.604,0.626] \cup[0.629,0.656]} & {[0.500,0.500]}
\end{array}\right)
\end{aligned}
$$

Finally, we have arrived to the last phase: Exploitation phase, where T-EWVM is applied. Before applying the algorithm some input values must be established: aggregation operator between finitely generated sets $\mathrm{Agg}$, order on finitely generated sets $\leq_{*}$ and optimism parameter $\alpha$.

In order to facilitate the comprehension of the method the easiest aggregation operator between finitely generated sets is chosen, that is, Agg is the arithmetic Mean. Furthermore, an $\alpha^{s g}$-point order $\leq_{*}=\leq_{0.5^{0}}$ is selected and the parameter is fixed in a quite pessimistic point of view $\alpha=0.25$. 
As our matrix $M$ is already normalized the first step of T-EWVM is unnecessary and we can continue to the second step, where positive preference $\left(\mathrm{P}^{+}\right)$ and negative preference are calculated:

$$
\begin{gathered}
P^{+}=\left(\begin{array}{cccc}
{[0,0]} & {[0,0.051]} & {[0,0.030]} & \emptyset \\
{[0,0.022] \cup[0.050,0.097]} & {[0,0]} & {[0.035,0.056]} & \emptyset \\
{[0,0.083]} & {[0.012,0.063]} & {[0,0]} & \emptyset \\
{[0.067,0.142] \cup[0.143,0.188]} & {[0.099,0.103] \cup[0.141,0.163]} & {[0.104,0.126] \cup[0.129,0.156]} & {[0,0]}
\end{array}\right) \\
P^{-}=\left(\begin{array}{ccccc}
{[0,0]} & {[-0.097,-0.050] \cup[-0.022,0]} & {[-0.083,0]} & {[-0.187,-0.143] \cup[-0.141,-0.067]} \\
{[-0.051,0]} & {[0,0]} & {[-0.062,-0.012]} & {[-0.162,-0.141] \cup[-0.103,-0.099]} \\
{[-0.030,0]} & {[-0.056,-0.035]} & {[0,0]} & {[-0.157,-0.129] \cup[-0.126,-0.104]} \\
\emptyset & \emptyset & \emptyset & {[0,0]}
\end{array}\right)
\end{gathered}
$$

Then, we can calculate $P^{0.25}=0.25 \cdot P^{+} \cup 0.75 \cdot P^{-}$:

$P^{0.25}=\left(\begin{array}{cccc}{[0,0]} & {[-0.073,-0.037] \cup[-0.017,0.013]} & {[-0.062,0.008]} & {[-0.140,-0.107] \cup[-0.106,-0.050]} \\ {[-0.038,0.006] \cup[0.012,0.024]} & {[0,0]} & {[-0.046,-0.009] \cup[0.009,0.014]} & {[-0.121,-0.105] \cup[-0.077,-0.074]} \\ {[-0.022,0.021]} & {[-0.042,-0.026] \cup[0.003,0.016]} & {[0,0]} & {[-0.117,-0.097] \cup[-0.094,-0.078]} \\ {[0.017,0.035] \cup[0.036,0.047]} & {[0.025,0.026] \cup[0.035,0.041]} & {[0.026,0.031] \cup[0.032,0.039]} & {[0,0]}\end{array}\right.$

Finally, we proceed with the assessment of $\mathcal{K}_{i}$. In the first loop these are the obtained indexes:

$$
\begin{aligned}
& I\left(O_{1}\right)=[-0.092,-0.057] \cup[-0.050,-0.010], \\
& I\left(O_{2}\right)=[-0.069,-0.036] \cup[-0.018,-0.012], \\
& I\left(O_{3}\right)=[-0.061,-0.041] \cup[-0.031,-0.014], \\
& I\left(O_{4}\right)=[0.023,0.031] \cup[0.035,0.042] .
\end{aligned}
$$

The greatest finitely generated set in relation to $\leq_{0.5^{\circ}}$ is $I\left(O_{4}\right)$ due to 0.031 is the greatest $0.5^{0}$-point. Since this moment, we do not consider the elements of $P^{0.25}$ related with $\mathrm{O}_{4}$ :

$$
P^{0.25}=\left(\begin{array}{ccccc}
{[0,0]} & {[-0.073,-0.037] \cup[-0.017,0.013]} & {[-0.062,0.008]} & {[-0.140,-0.107] \cup[-0.106,-0.050]} \\
{[-0.038,0.006] \cup[0.012,0.024]} & {[0,0]} & {[-0.046,-0.009] \cup[0.009,0.014]} & \frac{[-0.121,-0.105] \cup[-0.077,-0.074]}{[-0.117,-0.097] \cup[-0.094,-0.078]} \\
{[-0.022,0.021]} & {[-0.042,-0.026] \cup[0.003,0.016]} & {[0,0]} & {[0,-07}
\end{array} .\right.
$$

Then, in the second loop these are the obtained indexes:

$$
\begin{aligned}
& I\left(O_{1}\right)=[-0.068,-0.032] \cup[-0.022,0.010], \\
& I\left(O_{2}\right)=[-0.043,-0.002] \cup[0.011,0.019], \\
& I\left(O_{3}\right)=[-0.033,-0.014] \cup[0.001,0.018] .
\end{aligned}
$$

The greatest finitely generated set in relation to $\leq_{0.5^{0}}$ is now $I\left(\mathrm{O}_{2}\right)$ due to -0.002 is the greatest $0.5^{0}$-point. Since this moment, we do not consider the 
elements of $P^{0.25}$ related with $O_{2}$ and $O_{4}$ :

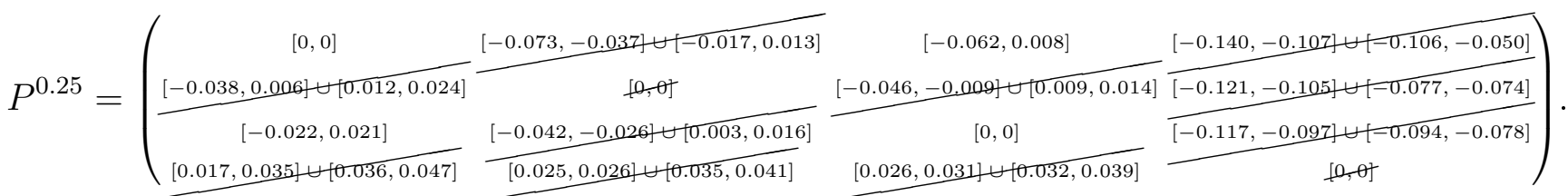

Conclusively, in the third loop these are the obtained indexes:

$$
\begin{aligned}
& I\left(O_{1}\right)=[-0.063,0.008], \\
& I\left(O_{3}\right)=[-0.023,0.021] .
\end{aligned}
$$

Finally, the greatest finitely generated set in relation to $\leq_{0.5^{0}}$ is now $I\left(O_{3}\right)$ due to -0.001 is the greatest $0.5^{0}$-point. Thus, the result of our group decision making problem is:

$$
O_{4}>O_{2}>O_{3}>O_{1} \text {. }
$$

\section{Conclusions and future research}

In this paper, the twofold group decision making problem was introduced. This approach to the group decision making problem allows to model at the same time influence of different experts and criteria.

In order to study this Twofold group decision making problem, several operations between non-negative finitely generated sets were defined. It is also shown an important theorem characterizing these operations in relation to certain $\alpha^{s g}$-points of the original sets. In addition, aggregation operators between finitely generated sets were introduced.

On the other hand, the steps required to resolve this new problem are established. Furthermore, in the Exploitation phase, EWVM is improved in order to work with this finite interval-valued hesitant fuzzy preference relations instead of common fuzzy preference relations. This improved version received the name of Twofold Extended Weighted Voting Method (T-EWVM). Finally, to illustrate how this twofold group decision making problem is resolved, a pedagogical example is shown.

In the future we will intend to improve the first step of a twofold group decision making problem. Some methods described in the joint phase could be improved in order to cope with outliers. Therefore, an improvement of this joint could be studied, for example using fuzzy sets instead of finitely generated sets or weighting the importance of the majority of the experts using some kind of 
penalty functions. However, a further study of these improvements is kept for future analysis.

\section{Acknowledgements}

The research reported in this paper has been supported by Project TIN201459543-P of the Spanish Ministry of Economy.

\section{References}

[1] J. C. Bezdek, D. Spliman, R. Spilman, A fuzzy relation space for group decision theory, Fuzzy Sets and Systems 1 (1978) 255-268.

[2] B. Bedregal, R. Reiser, H. Bustince, C. Lopez-Molina, V. Torra, Aggregation functions for typical hesitant fuzzy elements and the action of automorphisms, Information Sciences 255 (2014) 82-99.

[3] H. Bustince, A. Jurio, A. Pradera, R. Mesiar, G. Beliakov, Generalization of the weighted voting method using penalty functions constructed via faithful restricted dissimilarity functions, European Journal of Operational Research 225 (2013) 472-478.

[4] T. Calvo, G. Mayor, R. Mesiar, Aggregation operators: new trends and applications, Physica-Verlag, Heilderberg, New York, 2002.

[5] N. Chen, Z.S. Xu, M.M. Xia, Interval-valued hesitant preference relations and their applications to group decision making, Knowledge-Based Systems 37 (1) (2013) 528-540.

[6] F. Chiclana, F. Herrera, E. Herrera-Viedma, Integrating three representation models in fuzzy multipurpose decision making based on fuzzy preference relations, Fuzzy Sets and Systems 97 (1998) 33-48.

[7] D. Dubois, H. Prade, Ranking fuzzy numbers in the setting of possibility theory, Information theory 30 (1983) 183-224.

[8] Jànos Fodor, Marc Roubens, Fuzzy preference modelling and multicriteria decision support, Kluwer Academic Publishers, 1994.

[9] I. Grattan-Guinness, Fuzzy membership mapped onto interval and manyvalued quantities, Zeitschrift für mathematische Logik und Grundladen der Mathematik 22 (1976) 149-160.

[10] F. Herrera, L. Martínez, P. J. Sánchez, Managing non-homogeneous information in group decision making, European Journal of Operational Research 166 (2005) $115-132$. 
[11] E. Hüllermeier, K. Brinker, Learning valued preference structures for solving classification problems, Fuzzy Sets and Systems 159(18) (2008) 2337-2352.

[12] E. Hüllermeier, S. Vanderlooy, Combining predictions in pairwise classification: an optimal adaptive voting strategy and its relation to weighted voting, Pattern Recognition 43(1) (2010) 128-142.

[13] J.C. Hühn, E. Hüllermeier, FR3: a fuzzy rule learner for inducing reliable classifiers, IEEE Transactions on Fuzzy Systems 17(1) (2009) 138-149.

[14] V.S. Lai, B.K. Wong, W. Cheung, Group decision making in a multiple criteria environment: A case using the AHP in software selection, European Journal of Operational Research 137(1) (2002) 134-144.

[15] H.C. Liao, Z.S. Xu, X.J. Zeng, J.M. Merigó, Qualitative decision making with correlation coefficients of hesitant fuzzy linguistic term sets, Knowledge-Based Systems 76 (2015) 127-138.

[16] K. Nakamura, Preference relation on a set of fuzzy utilities as a basis for decision making, Fuzzy Sets and Systems 20 (1986) 147-162.

[17] I.J. Pérez, S. Alonso, F.J. Cabrerizo, J. Lu, E. Herrera-Viedma, Modelling Heterogeneity among Experts in Multi-criteria Group Decision Making Problems, Lecture Notes in Computer Science 6820 (2011) 55-66.

[18] R. Pérez-Fernández, P. Alonso, I. Díaz, S. Montes, Multi-Factorial Risk Assessment: An Approach based on Fuzzy Preference Relations, Fuzzy Sets and Systems, in press. DOI: 10.1016/j.fss.2014.10.012.

[19] R. Pérez-Fernández, P. Alonso, H. Bustince, I. Díaz, A. Jurio, S. Montes, Ordering finitely generated sets and finite interval-valued hesitant fuzzy sets. Submitted.

[20] T. Rashid, S.M. Husnine, Multicriteria Group Decision Making by Using Trapezoidal Valued Hesitant Fuzzy Sets, The Scientific World Journal, 2014, Article ID 304834, 2014. doi:10.1155/2014/304834.

[21] R.M. Rodríguez, L. Martínez, V. Torra, Z.S. Xu and F. Herrera, Hesitant fuzzy sets: State of the art and future directions, International Journal of Intelligent Systems 29(6) (2014) 495-525.

[22] M. Tavana, CROSS: A Multicriteria Group-Decision-Making Model for Evaluating and Prioritizing Advanced-Technology Projects at NASA, Interfaces 33(3) (2003) 40-56.

[23] V. Torra, Hesitant fuzzy sets, International Journal of Intelligent Systems 25 (2010) 529-539.

[24] J. Wang, Z. Han, H. Zhang, Multi-criteria Group Decision-Making Method Based on Intuitionistic Interval Fuzzy Information, Group Decision and Negotiation 23(4) (2014) 715-733.

[25] Z.S. Xu, Hesitant Fuzzy Sets Theory, Springer, Berlin, 2014. 
[26] D. Yu, W. Zhang, Y. Xu, Group decision making under hesitant fuzzy environment with application to personnel evaluation, Knowledge-Based Systems 52 (2013) 1-10.

[27] D. Yu, Some Hesitant Fuzzy Information Aggregation Operators Based on Einstein Operational Laws, International Journal of Intelligent Systems 29(4) (2014) 320-340.

[28] Y. Yuan, Criteria for evaluating fuzzy ranking methods, Fuzzy Sets and Systems 43 (1991) 139-157. 